\title{
Second-Order Approximate Symmetries of the Geodesic Equations for the Reissner-Nordström Metric and Re-Scaling of Energy of a Test Particle ${ }^{\star}$
}

\author{
Ibrar HUSSAIN ${ }^{\dagger}$, Fazal M. MAHOMED ${ }^{\ddagger}$ and Asghar QADIR ${ }^{\dagger}$ \\ $\dagger$ Centre for Advanced Math. and Phys., National University of Sciences and Technology, \\ Campus of the College of Electr. and Mech. Eng., Peshawar Road, Rawalpindi, Pakistan \\ E-mail: ihussain@camp.edu.pk, aqadirmath@yahoo.com \\ $\ddagger$ School of Computational and Applied Mathematics, University of the Witwatersrand, \\ Wits 2050, South Africa \\ E-mail: Fazal.Mahomed@wits.as.za
}

Received August 14, 2007, in final form November 16, 2007; Published online December 07, 2007

Original article is available at http://www.emis.de/journals/SIGMA/2007/115/

\begin{abstract}
Following the use of approximate symmetries for the Schwarzschild spacetime by A.H. Kara, F.M. Mahomed and A. Qadir (Nonlinear Dynam., to appear), we have investigated the exact and approximate symmetries of the system of geodesic equations for the Reissner-Nordström spacetime (RN). For this purpose we are forced to use second order approximate symmetries. It is shown that in the second-order approximation, energy must be rescaled for the RN metric. The implications of this rescaling are discussed.
\end{abstract}

Key words: Reissner-Nordström metric; geodesic equations; second-order approximate symmetries

2000 Mathematics Subject Classification: 83C40; 70 S10

\section{Introduction}

Though most of the well known spacetimes of General Relativity (GR) are time-translational invariant, generally this is not guaranteed [2]. Consequently energy is not generally conserved and hence there is no good definition for energy [3]. Nowhere is this problem faced as severely as in gravitational wave spacetimes, where energy is not given by the stress-energy tensor, which is zero [2]. For these spacetimes one can check that they impart energy to test particles in their path $[4,5]$ despite the fact that the stress-energy tensor is zero. The lack of a good definition of energy, also leads to problems with the definition of mass [3]. As such, one needs to use some concept of time symmetry that allows for slight deviations away from exact symmetry.

This approach was attempted earlier by various people. There have been a number of different definitions of "approximate symmetry". One idea was to assume that conservation of energy holds asymptotically [6] and to examine whether it would work for gravitational radiation and to define a positive definite energy. This seems unsatisfactory as the gravitational energy should reach (the place) infinity. There may then be problems with orders of approximation being consistent. An altogether different approach was taken by providing a measure of the extent of break-down of symmetry. The integral of the square of the symmetrized derivative of a vector field was divided by its mean square norm [7,8]. This led to what was called an almost symmetric space and the corresponding vector field an almost Killing vector [9]. This

\footnotetext{
${ }^{\star}$ This paper is a contribution to the Proceedings of the Seventh International Conference "Symmetry in Nonlinear Mathematical Physics" (June 24-30, 2007, Kyiv, Ukraine). The full collection is available at http://www.emis.de/journals/SIGMA/symmetry2007.html
} 
measure of "non-symmetry" in a given direction was applied to the Taub cosmological solution [10] and to study gravitational radiation. It provides a choice of gauge that makes calculations simpler and was used for this purpose [11]. Essentially based on the almost symmetry, the concept of an "approximate symmetry group" was presented [12]. However it has not been unequivocally successful either. The approach of a slightly broken symmetry seems promising, but merely providing simplicity of calculations is not physically convincing. Other approaches need to be tried, to find one that seems significantly better than others.

The development of geometry has been driven by its application to kinematics and dynamics. As such, one might look at a geometrically driven definition of symmetry. On the other hand, we are concerned with conservation laws, which are given by the invariants of the EulerLagrange equations. Hence, for our purposes, Lie symmetries as embodied in isometries and in Noether's theorem, should provide us the desired approach to define "approximate symmetries". Now, there is a connection between isometries and the symmetries of differential equations (DEs) through the geodesic equations $[13,14]$. We propose that the Baikov-GazizovIbragimov concept of "approximate symmetry" $[15,16]$ could be extended and adapted for the purpose of defining energy in GR via "approximate isometries" through the above-mentioned connection between the symmetries of geometry and the symmetries of differential equations. Using that connection approximate symmetries of the Schwarzschild metric were discussed [1]. The Schwarzschild metric has much fewer symmetries than the Minkowski metric (only 4 generators). One would expect that in the limit of small gravitational mass the "lost" symmetries should be "approximately" recovered. Of course, this is a static metric and energy is conserved and it is not relevant for a discussion of energy. However, linear and spin angular momentum conservation is lost. The "trivial" approximate symmetries of the geodesic equations recover these as approximately conserved quantities. To be able to interpret the approximate symmetry results so obtained we need to first follow how higher order approximate symmetries are to be understood for our purpose and then how the lack of time-translational symmetry on a globally defined spacelike hypersurface is to be interpreted. As such, before dealing with gravitational waves it is necessary to study the Reissner-Nordström (RN) metric and the Kerr metric from this point of view. In this paper we present the discussion of $\mathrm{RN}$ spacetime leaving the other spacetimes for subsequent consideration.

In going to the RN spacetime the approximate symmetries and conservation laws, which were recovered in the first-order approximation [1], are lost. Nor is there any approximate symmetry for the reduced orbital equation. However one would expect that in the limit of small charge we should recover the lost symmetries. To recover the conservation laws lost again we need to appeal to second-order approximate symmetries. It makes no difference whether the exact or perturbed equation is used in the definition of first-order approximate symmetries. In this paper it is shown that in the definition of second-order approximate symmetries it makes a significant difference. We use the perturbed equation in the definition of second-order approximate symmetries, the expression for energy of a test particle in the RN metric is re-scaled.

The plan of the paper is as follows. In the next section we explain the concept of symmetry and approximate symmetry of DEs, and approximate symmetries of the Schwarzschild metric. In Section 3 approximate symmetries of the orbital equation for the RN metric and of the system of geodesic equations for this metric are discussed. Finally a summary and discussion is given in Section 4.

\section{Symmetries and approximate symmetries of DEs}

Symmetries are very useful because they are directly connected to the conservation laws through Noether's theorem [17, 18, 19]. If, for a given system of DEs there is a variational principle, then a continuous symmetry under which the action functional remains invariant yields a con- 
servation law $[20,21,22,23]$. Here we are interested in Lie symmetry methods [24]. The set of infinitesimal symmetry generators of a system of differential equations form a Lie algebra $[21,25,16]$. Geometrically the symmetries of a manifold are characterized by its Killing vectors, or isometries, which also form a Lie algebra [26]. The symmetries of the manifold are inherited by the geodesic equations on it with additional symmetries [14]. They give quantities conserved under geodesic motion [27] and lead to first integrals of the geodesic equations [28].

It may often happen that a manifold does not possess exact symmetry but approximately does so. We may be able to obtain more information from near symmetry or broken symmetry, than from the exact symmetry always maintained. As such approximate symmetries of a manifold are worth exploring. Methods for obtaining the approximate symmetries of a system of ordinary differential equations (ODEs) are available in the literature [29, 15].

A system of $p$ ODEs of order $n[25,16]$

$$
\mathbf{E}\left(s ; \mathbf{x}(s), \mathbf{x}^{\prime}(s), \mathbf{x}^{\prime \prime}(s), \ldots, \mathbf{x}^{(n)}(s)\right)=0,
$$

has the symmetry generator

$$
\mathbf{X}^{[n]}=\xi(s, \mathbf{x}) \frac{\partial}{\partial s}+\eta^{\alpha}(s, \mathbf{x}) \frac{\partial}{\partial x^{\alpha}}+\eta_{, s}^{\alpha}\left(s, \mathbf{x}, \mathbf{x}^{\prime}\right) \frac{\partial}{\partial x^{\alpha \prime}}+\cdots+\eta_{,(n)}^{\alpha}\left(s, \mathbf{x}, \mathbf{x}^{\prime}, \ldots, \mathbf{x}^{(n)}\right) \frac{\partial}{\partial x^{\alpha(k)}},
$$

if $\mathbf{X}^{[n]}$ annihilates the system of equations $\mathbf{E}$ (for solutions of the equation)

$$
\left.\mathbf{X}^{[n]}(\mathbf{E})\right|_{\mathbf{E}=0}=0,
$$

where $\mathbf{x}$ is a point in the underlying $n$-dimensional space and $\mathbf{x}^{(n)}$ is the $n$th derivative with respect to $s$. The prolongation coefficients are given by

$$
\eta_{,(n)}^{\alpha}=\frac{d \eta_{,(n-1)}^{\alpha}}{d s}-x^{\alpha(n)} \frac{d \xi}{d s}, \quad n \geq 1, \quad \text { where } \quad \eta_{,(1)}^{\alpha}=\eta_{, s}^{\alpha} .
$$

If $p=1$ then the system reduces to a single equation.

The $k$ th-order approximate symmetry of a perturbed system of ODEs

$$
\mathbf{E}=\mathbf{E}_{0}+\epsilon \mathbf{E}_{1}+\epsilon^{2} \mathbf{E}_{2}+\cdots+\epsilon^{k} \mathbf{E}_{k}+O\left(\epsilon^{k+1}\right)
$$

is given by the generator

$$
\mathbf{X}=\mathbf{X}_{0}+\epsilon \mathbf{X}_{1}+\epsilon^{2} \mathbf{X}_{2}+\cdots+\epsilon^{k} \mathbf{X}_{k},
$$

if the symmetry condition

$$
\mathbf{X E}:=\left[\left(\mathbf{X}_{0}+\epsilon \mathbf{X}_{1}+\epsilon^{2} \mathbf{X}_{2}+\cdots+\epsilon^{k} \mathbf{X}_{k}\right)\left(\mathbf{E}_{0}+\epsilon \mathbf{E}_{1}+\epsilon^{2} \mathbf{E}_{2}+\cdots+\epsilon^{k} \mathbf{E}_{k}\right)\right]_{(2)}=O\left(\epsilon^{k+1}\right)
$$

is satisfied [19, 29, 30]. Here $\mathbf{E}_{0}$ is the exact system of equations, $\mathbf{E}_{1}$ is the first-order approximate part and $\mathbf{E}_{2}$ is the second-order approximate part of the perturbed system and so on; $\mathbf{X}_{0}$ is the exact symmetry generator, $\mathbf{X}_{1}$ the first-order approximate part, $\mathbf{X}_{2}$ the second-order approximate part of the symmetry generator and so on. These approximate symmetries do not necessarily form a Lie algebra but do form a so-called "approximate Lie algebra" [31].

If (2) admits (3) with $\mathbf{X}_{0} \neq 0$, then $\mathbf{X}_{0}$ is an exact symmetry of the unperturbed $\mathbf{E}_{0}=0$ and $\mathbf{X}_{0}$ is a stable symmetry for a given perturbation. The perturbed equation (2) always has the approximate symmetry $\epsilon \mathbf{X}_{0}$ which is known as a trivial symmetry and $\mathbf{X}$ given by (3) with $\mathbf{X}_{0} \neq 0$ and $\mathbf{X}_{1} \neq \mathbf{X}_{0}$ is called a non-trivial approximate symmetry [32].

An alternate method for defining approximate symmetries of DEs was given by Fushchich and Shtelen [33]. They interchange the order of approximation and take the limit, between the parameter of the symmetry generator of the algebra on one hand and the approximation parameter on the other hand. This method is compared with that of Baikov et al. in [34, 35]. For our purpose it makes no difference which of these methods is used. We will follow the method of Baikov et al. [15]. 


\subsection{Symmetries and approximate symmetries of the Schwarzschild metric}

Minkowski spacetime has the Poincaré symmetry algebra $s o(1,3) \oplus_{s} \mathbb{R}^{4}$ (where $\oplus_{s}$ denotes semi direct sum) with the 10 generators [36, 2]

$$
\begin{aligned}
& \mathbf{X}_{0}=\frac{\partial}{\partial t}, \quad \mathbf{X}_{1}=\cos \phi \frac{\partial}{\partial \theta}-\cot \theta \sin \phi \frac{\partial}{\partial \phi}, \\
& \mathbf{X}_{2}=\sin \phi \frac{\partial}{\partial \theta}+\cot \theta \cos \phi \frac{\partial}{\partial \phi}, \quad \mathbf{X}_{3}=\frac{\partial}{\partial \phi}, \\
& \mathbf{X}_{4}=\sin \theta \cos \phi \frac{\partial}{\partial r}+\frac{\cos \theta \cos \phi}{r} \frac{\partial}{\partial \theta}-\frac{\csc \theta \sin \phi}{r} \frac{\partial}{\partial \phi}, \\
& \mathbf{X}_{5}=\sin \theta \sin \phi \frac{\partial}{\partial r}+\frac{\cos \theta \sin \phi}{r} \frac{\partial}{\partial \theta}+\frac{\csc \theta \cos \phi}{r} \frac{\partial}{\partial \phi}, \\
& \mathbf{X}_{6}=\cos \theta \frac{\partial}{\partial r}-\frac{\sin \theta}{r} \frac{\partial}{\partial \theta}, \\
& \mathbf{X}_{7}=\frac{r \sin \theta \cos \phi}{c} \frac{\partial}{\partial t}+c t\left(\sin \theta \cos \phi \frac{\partial}{\partial r}+\frac{\cos \theta \cos \phi}{r} \frac{\partial}{\partial \theta}-\frac{\csc \theta \sin \phi}{r} \frac{\partial}{\partial \phi}\right), \\
& \mathbf{X}_{8}=\frac{r \sin \theta \sin \phi}{c} \frac{\partial}{\partial t}+c t\left(\sin \theta \sin \phi \frac{\partial}{\partial r}+\frac{\cos \theta \sin \phi}{r} \frac{\partial}{\partial \theta}+\frac{\csc \theta \cos \phi}{r} \frac{\partial}{\partial \phi}\right), \\
& \mathbf{X}_{9}=\frac{r \cos \theta}{c} \frac{\partial}{\partial t}+c t\left(\cos \theta \frac{\partial}{\partial r}-\frac{\sin \theta}{r} \frac{\partial}{\partial \theta}\right),
\end{aligned}
$$

$\mathbf{X}_{0}, \mathbf{X}_{4}, \mathbf{X}_{5}$ and $\mathbf{X}_{6}$ are the spacetime translations (with a Lie sub-algebra $\left(\mathbb{R}^{4}\right)$ ) which provide the laws of conservation of energy and linear momentum, $\mathbf{X}_{1}, \mathbf{X}_{2}$ and $\mathbf{X}_{3}$ are the rotations (with a Lie sub-algebra $(s o(3))$ ) which provide the laws of conservation of angular momentum and $\mathbf{X}_{7}, \mathbf{X}_{8}$ and $\mathbf{X}_{9}$ are the Lorentz transformations (with a Lie sub-algebra (so(3))) which provide the laws of conservation of spin angular momentum; via Noether's theorem [17]. The symmetry algebra for the geodesic equations is $\operatorname{sl}(6, \mathbb{R})$, which has many symmetries that do not correspond to conservation laws [14].

For the Schwarzschild metric, corresponding to a point mass, $M$, situated at the origin only the first 4 generators $\left(\mathbf{X}_{0}, \mathbf{X}_{1}, \mathbf{X}_{2}, \mathbf{X}_{3}\right)$ apply, yielding only conservation of energy and angular momentum. The generators $\mathbf{X}_{5}, \ldots, \mathbf{X}_{9}$, yielding conservation of linear momentum and spin angular momentum are "lost". Using the approximate symmetries for the system of geodesic equations for this metric with $\epsilon=2 G M / c^{2}$ [1] (where $G$ is Newton's gravitational constant and $c$ is the speed of light in vacuum) the "lost" symmetries are recovered as trivial approximate symmetries yielding approximate conservation of those quantities. The symmetry algebra of the geodesic equations for the Schwarzschild metric is $s o(3) \oplus \mathbb{R} \oplus d_{2}$ (where $d_{2}$ is the dilation algebra with generators $\partial / \partial s$ and $s \partial / \partial s$ ) [14]. The geodesic equations can be reduced to a single orbital equation that has the two non-trivial approximate symmetries given by the generators

$$
\begin{aligned}
& \mathbf{X}_{a 1}=\sin \phi \frac{\partial}{\partial u}+\epsilon\left(2 \sin \phi \frac{\partial}{\partial \phi}+u \cos \phi \frac{\partial}{\partial u}\right) \\
& \mathbf{X}_{a 2}=\cos \phi \frac{\partial}{\partial u}-\epsilon\left(2 \cos \phi \frac{\partial}{\partial \phi}-u \sin \phi \frac{\partial}{\partial u}\right) .
\end{aligned}
$$

\section{Symmetries and approximate symmetries of the Reissner-Nordström metric}

The field of a point massive electric charge at rest at the origin is given by the RN metric (see for example [37])

$$
d s^{2}=e^{\nu} d t^{2}-e^{-\nu} d r^{2}-r^{2} d \theta^{2}-r^{2} \sin ^{2} \theta d \phi^{2},
$$


with

$$
e^{\nu}=1-\frac{2 G M}{c^{2} r}+\frac{G Q^{2}}{c^{4} r^{2}},
$$

where $Q$ is the electric charge of the point gravitational source. Electromagnetism is the only long range force in Nature other than gravity and this is the only spherically symmetric, static exact solution of the "sourceless" Einstein-Maxwell equations. In the chargeless case $(Q=0)$ it reduces to the Schwarzschild metric. It is of interest to look at the symmetry structure of this metric and the corresponding symmetries, and approximate symmetries of the geodesic equations.

It had been pointed out [1] that there is a difference between the conservation laws obtained for the system of geodesic equations and the single, orbital equation for the Schwarzschild metric. It was further remarked that it should be checked if this difference also holds for other spacetimes. We investigate this question for the orbital equation in the RN metric,

$$
\frac{d^{2} u}{d \phi^{2}}+u=\frac{G M}{h^{2}}-\frac{G Q^{2}}{c^{2} h^{2}} u+\frac{3 G M}{c^{2}} u^{2}-\frac{2 G Q^{2}}{c^{2}} u^{3},
$$

where $h$ is the classical angular momentum per unit mass and $u=1 / r$. In the classical limit $c \rightarrow \infty$ it gives the classical orbital equation and for $Q=0$ it yields the Schwarzschild orbital equation. For the approximate symmetries of this equation we take

$$
\epsilon=\frac{2 G M}{c^{2}}, \quad \frac{G Q^{2}}{c^{4}}=k \epsilon^{2} .
$$

For an RN black hole (see for example [38]) that is

$$
M^{2} \geq Q^{2}, \quad \text { we have } 0<k \leq 1 / 4 \text {. }
$$

If $k>1 / 4$ the metric represents a naked singularity. Taking $\epsilon=0$ in the orbital equation, the exact symmetry generators are

$$
\begin{array}{ll}
\mathbf{X}_{0}=u \frac{\partial}{\partial u}, \quad \mathbf{X}_{1}=\cos \phi \frac{\partial}{\partial u}, & \mathbf{X}_{2}=\sin \phi \frac{\partial}{\partial u}, \\
\mathbf{X}_{4}=\cos 2 \phi \frac{\partial}{\partial \phi}-u \sin 2 \phi \frac{\partial}{\partial u}, & \mathbf{X}_{3}=\frac{\partial}{\partial \phi} \\
\mathbf{X}_{6}=u \cos \phi \frac{\partial}{\partial \phi}-u^{2} \sin \phi \frac{\partial}{\partial u}, & \mathbf{X}_{7}=u \sin \phi \frac{\partial}{\partial \phi}+u \cos 2 \phi \frac{\partial}{\partial u}
\end{array}
$$

Retaining terms of first-order in $\epsilon$ and neglecting $O\left(\epsilon^{2}\right)$, the first approximate symmetry generators are given by (13). In the second approximation, that is when we retain terms quadratic in $\epsilon$, this equation possesses no non-trivial second-order approximate symmetry generators, but the first-order approximate symmetry generators are still retained. Thus there is no new approximate conservation laws but only the previous conservation laws that have been recovered.

A better idea of what is actually required comes from the full system of geodesic equations. The geodesic equations are given by

$$
\begin{aligned}
& \ddot{t}+\nu^{\prime} \dot{t} \dot{r}=0, \\
& \ddot{r}+\frac{1}{2}\left(e^{\nu}\right)^{\prime}\left(e^{\nu} c^{2} \dot{t}^{2}-e^{-\nu} \dot{r}^{2}\right)-r e^{\nu}\left(\dot{\theta}^{2}+\sin ^{2} \theta \dot{\phi}^{2}\right)=0, \\
& \ddot{\theta}+\frac{2}{r} \dot{r} \dot{\theta}-\sin \theta \cos \theta \dot{\phi}^{2}=0, \\
& \ddot{\phi}+\frac{2}{r} \dot{r} \dot{\phi}+2 \cot \theta \dot{\theta} \dot{\phi}=0,
\end{aligned}
$$


with $e^{\nu}$ now given by (14) and hence

$$
\nu^{\prime}=\frac{\epsilon}{r^{2}}+\frac{1-2 k}{r^{3}} \epsilon^{2}, \quad\left(e^{\nu}\right)^{\prime}=\frac{\epsilon}{r^{2}}-\frac{2 k}{r^{3}} \epsilon^{2}, \quad e^{-\nu}=1+\frac{\epsilon}{r}+\frac{(1-k)}{r^{2}} \epsilon^{2},
$$

where "." denotes differentiation with respect to the geodetic parameter $s$.

To construct the determining equations for the second-order approximate symmetries we use (5) and (6) as the 4 exact symmetry generators and (7)-(12) as the 6 first-order approximate symmetry generators. Of the 4 exact generators 2 do not appear in the new determining equations and the other 2 cancel out. The 6 generators of the first-order approximate symmetry have to be eliminated for consistency of the new determining equations, making them homogeneous. The resulting system is the same as for Minkowski spacetime, yielding 10 second-order approximate symmetry generators. Four of them are again the exact symmetry generators used earlier, and hence simply "add into" them, making no difference. The other 6 replace the lost first-order approximate symmetry generators. The full set has the Poincaré algebra $s o(1,3) \oplus_{s} \mathbb{R}^{4}$ apart from some non-Noether symmetries. There are no non-trivial second-order approximate symmetries as was the case for the first-order approximate symmetries.

It is worth remarking that for the first-order approximate symmetries it did not matter whether we used the full system (2) or the un-perturbed system $\mathbf{E}_{0}$, in (4). However, for the second-order approximate symmetries it does make a difference. One needs to use the full system (2) and not the un-perturbed system $\mathbf{E}_{0}$, in (4) to obtain the solution.

The exact symmetry generators include not only (5) and (6), but also the generators of the dilation algebra, $\partial / \partial s, s \partial / \partial s$ corresponding to

$$
\xi(s)=c_{0} s+c_{1} .
$$

In the determining equations for the first-order approximate symmetries the terms involving $\xi_{s}=c_{0}$ cancel out. However, for the second-order approximate symmetries the terms in $\xi_{s}$ do not automatically cancel out but collect a scaling factor of $(1-2 k)$ so as to cancel out. (This factor comes from the application of the perturbed system, rather than the un-perturbed one.) Since energy conservation comes from time translational invariance and $\xi$ is the coefficient of $\partial / \partial s$ in the point transformations given by $(1)$, where $s$ is the proper time, the scaling factor $(1-2 k)$ corresponds to a re-scaling of energy. Thus, whereas there was no energy re-scaling needed for the first-order approximate symmetries, it arises naturally in the second-order approximate symmetry. Using (15) we get the energy re-scaling factor

$$
(1-2 k)=\left(1-Q^{2} / 2 G M^{2}\right) \text {. }
$$

Thus, even though there are no non-trivial second-order approximate symmetries, we get the non-trivial result of energy re-scaling from the second-order approximation. This point will be discussed further in the next section.

It is worth remarking that when some symmetries are "lost" at one order (exact or first-order approximate) they are "recovered" at the next (at least to second-order) as "trivial" approximate symmetries.

\section{Summary and discussion}

We studied the approximate symmetries of the RN metric. This metric has isometry algebra $s o(3) \oplus \mathbb{R}$ with generators (5) and (6). The symmetry algebra of the geodesic equations for this metric is $s o(3) \oplus \mathbb{R} \oplus d_{2}$. We used Lie symmetry methods for DEs to explore the second-order approximate symmetries of the RN metric. Neglecting terms containing $\epsilon^{2}$ in the geodesic equations (16) this metric has the same first approximate symmetries as those of the Schwarzschild 
metric. Again we get no non-trivial approximate symmetry generator in the second approximation. We only recover the "lost" conservation laws as approximate conservation laws. As for the Schwarzschild metric, where there is a difference between the conservation laws obtained for the system of geodesic equations and for the single orbital equation, the difference also holds for the RN metric.

The re-scaling of energy for the RN metric, which does not appear for the Schwarzschild metric is of special interest. Notice that the pseudo-Newtonian formalism [39, 40, 41, 42] gives re-scaling of force by $\left(1-Q^{2} / r M c^{2}\right)$. The reduction is by the ratio of the electromagnetic potential energy at a distance $r$ to the rest energy of the gravitational source. It is position dependent. That would not be reasonable for the energy in the field by itself. The scaling obtained here, $\left(1-Q^{2} / 2 G M^{2}\right)$, is more reasonable as relating the electromagnetic self-energy to the gravitational self-energy, with the radial parameter, $r$, canceled out.

It would be of interest to apply the definition of second-order approximate symmetries to the Kerr metric where there are only two isometries $\mathbf{X}_{0}=\partial / \partial t, \mathbf{X}_{3}=\partial / \partial \phi$ and a non-trivial Killing tensor [2] to see if the same result of energy re-scaling holds. It would also be important to check if the energy in the gravitational field could be obtained by considering approximate symmetries of gravitational wave spacetimes. For this purpose one can first consider some nonflat static spacetime. Since gravitational wave spacetimes are non-static solutions of the vacuum Einstein field equations (EFEs), one should perturb the static spacetime with a time dependent small parameter and then look at the approximate symmetries of this gravitational wave-like spacetime. As gravitational wave spacetimes are solutions of vacuum EFEs, one would have to calculate the Weyl and stress-energy tensor for this gravitational wave-like spacetime (for which the stress-energy tensor is non-zero) to equal orders of approximation to see how much energy is contained in the gravitational field and how much in the matter field. To understand the actual problem one will then have consider an exact gravitational wave solution and look at its approximate symmetries. For the exact gravitational wave solutions a formula for the momentum imported to test particle is already available [43]. The comparison of the two results might enable one to identify a physically significant energy content in the gravitational wave spacetime.

\section{Acknowledgements}

IH would like to thank Pakistan Science Foundation (PSF) for their full financial support to attend the Seventh International Conference "Symmetry in Nonlinear Mathematical Physics", where this work was presented. AQ would like to thank the conference organizers for the local hospitality and International Mathematical Union (IMU) for the traveling grant and DECMA and the School for Computational and Applied Mathematics of the University of Witwatersrand, Johannesberg, where the writing-up was completed.

\section{References}

[1] Kara A.H., Mahomed F.M., Qadir A., Approximate symmetries and conservation laws of the geodesic equations for the Schwarzschild metric, Nonlinear Dynam., to appear.

[2] Kramer D., Stephani H., MacCullum M.A.H., Herlt E., Exact solutions of Einstein field equations, Cambridge University Press, Cambridge, 1980.

[3] Landau L.D., Lifshitz E.M., The classical theory of fields, Pergamon Press, 1975.

[4] Weber J., Wheeler J.A., Reality of the cylindrical gravitational waves of Einstein and Rosen, Rev. Modern Phys. 29 (1957), 509-515.

[5] Ehlers J., Kundt W., Exact solutions of gravitational field equations, in Gravitation: An Introductoion to Current Research, Editor L. Witten, Wiley, New York, 1962, 49-101. 
[6] Komar A., Asymtotic covariant conservation laws for gravitational radiation, Phys. Rev. 127 (1962), 14111418.

Komar A., Positive-definite energy density and global consequences for general relativity, Phys. Rev. 129 (1963), 1873-1876.

[7] Matzner R., Almost symmetric spaces and gravitational radiation, J. Math. Phys. 9 (1968), 1657-1668. Matzner R., 3-sphere "backgrounds" for the space section of the Taub cosmological solution, J. Math. Phys. 9 (1968), 1063-1067.

[8] Isaacson R.A., Gravitational radiation in the limit of high frequency. I. The linear approximation and geometric potics, Phys. Rev. 166 (1968), 1263-1271.

Isaacson R.A., Gravitational radiation in the limit of high frequency. II. Non-linear terms and the effective stress tensor, Phys. Rev. 166 (1968), 1272-1280.

[9] York J.W., Covariant decomposition of symmetric tensor in the theory of gravitation, Ann. Inst. H. Poincaré Sect. A (N.S.) 21 (1974), 319-332.

[10] Taub A.H., Empty spacetime admitting a three parameter groups of motions, Ann. Math. 53 (1951), 472490.

[11] Bona C., Carot J., Palenzueala-Luque C., Almost-stationary motions and gauge conditions in general relativity, Phys. Rev. D $\mathbf{7 2}$ (2005), 124010, 5 pages, gr-qc/0509015.

[12] Spero A., Baierlein R., Approximate symmetry groups of inhomogeneous metrics, J. Math. Phys. 18 (1977), 1330-1340.

Spero A., Baierlein R., Approximate symmetry groups of inhomogeneous metrics: examples, J. Math. Phys. 19 (1978), 1324-1334.

[13] Aminova A.V., Projective transformations and symmetries of differential equations, Sb. Math. 186 (1995), $1711-1726$.

[14] Feroze T., Mahomed F.M., Qadir A., The connection between isometries and symmetries of the geodesic equations of the underlying spaces, Nonlinear Dynam. 45 (2006), 65-74.

[15] Baikov V., Gazizov R.K., Ibragimov N.H., Approximate symmetries of equations with small parameters, Mat. Sb. 136 (1988), 435-450 (English transl.: Math. USSR Sb. 64 (1989), 427-441).

[16] Ibragimov N.H., Elementary Lie group analysis and ordinary differential equations, Wiley, Chichester, 1999.

[17] Noether E., Invariant variations problems, Nachr. Konig. Gissell. Wissen., Gottingen, Math.-Phys. Kl. 2 (1918), 235-257 (English transl.: Transport Theory and Stat. Phys. 1 (1971), 186-207).

[18] Bluman G., Connections between symmetries and conservation laws, SIGMA 1 (2005), 011, 16 pages, math-ph/0511035.

[19] Kara A.H., Mahomed F.M., A basis of conservation laws for partial differential equations, J. Nonlinear Math. Phys. 9 (2002), 60-72.

[20] Bessel-Hagen E., Über die Erhaltungssätze der Elektrodynamik, Math. Ann. 84 (1921), 258-276.

[21] Ibragimov N.H., Transformation groups applied to mathematical physics, Reidel, Boston, 1985.

[22] Olver P.J., Applications of Lie groups to differential equations, Springer-Verlag, New York, 1993.

[23] Bluman G., Kumei S., Symmetries and differential equations, Springer-Verlarg, New York, 1989.

[24] Lie S., Sophus Lie's 1880 transformation group paper, Math Sci. Press, Brookline MA, 1975 (translated by Michael Ackerman. Comments by Robert Hermann).

[25] Stephani H., Differential equations: their solutions using symmetry, Cambridge University Press, New York, 1989.

[26] Hawking S.W., Ellis G.F.R., The large scale structure of spacetime, Cambridge University Press, Cambridge, 1973.

[27] Caviglia G., Dynamical symmetries: an approach to Jacobi fields and to constants of geodesic motion, J. Math. Phys. 24 (1983), 2065-2069.

[28] Katzin G.H., Levine J., Geodesic first integral with explicit path-parameter dependence in Riemannian spacetime, J. Math. Phys. 22 (1981), 1878-1891.

[29] Baikov V., Gazizov R.K., Ibragimov N.H., Differential equations with a small parameter: exact and approximate symmetries, in CRC Handbook of Lie Group Analysis of Differential Equations, Vol. 3, Editor N.H. Ibragimov, CRC Press, Boca Raton, Florida, 1996, 217-282.

[30] Kara A.H., Mahomed F.M., Ünal G., Approximate symmetries and conservation laws with applications, Int. J. Theor. Phys. 38 (1999), 2389-2399. 
[31] Gazizov R.K., Lie algebras of approximate symmetries, J. Nonlinear Math. Phys. 3 (1996), 96-101.

[32] Baikov V., Gazizov R.K., Ibragimov N.H., Mahomed F.M., Closed orbits and their stable symmetries, J. Math. Phys. 35 (1994), 6525-6535.

[33] Fushchich W.I., Shtelen W.M., On approximate symmetry and approximate solutions of the non-linear wave equation with a small parameter, J. Phys. A: Math. Gen. 22 (1989), L887-L890.

[34] Pakdemirli M., Yürüsoy M., Dolapci I., Comparison of approximate symmetry methods for differential equatons, Acta Appl Math. 80 (2004), 243-271.

[35] Valenti A., Approximate symmetries for a model describing dissipative media, in Proceedings of 10th International Conference in Modern Group Analysis (MOGRAN X) (October 24-31, 2004, Larnaca, Cyprus), Editors N.H. Ibrahimov, C. Sophocleous and P.A. Damianou, 2005, 236-243.

[36] Hall G.S., Symmetries and curvature structure in general relativity, World Scientific, Singapore, 2004.

[37] Misner C.W., Thorne K.S., Wheele J.A., Gravitation, W.H. Freeman and Company, San Francisco, 1973.

[38] Inverno D.R., Introducing Einstein's relativity, Oxford University Press, New York, 1992.

[39] Qadir A., General relativity in terms of forces, in Proceedings of Third Regional Conference on Mathematical Physics, Editors F. Hussain and A. Qadir, World Scientific, 1990, 481-490.

[40] Qadir A., The gravitational force in general relativity, in M.A.B. Beg Memorial Volume, Editors A. Ali and P. Hoodbhoy, World Scientific, 1991, 159-178.

[41] Qadir A., Sharif M., The relativistic generalization of the gravitational force for arbitary spacetimes, Nuovo Cimento B 117 (1992), 1071-1083.

[42] Qadir A., Zafarullah I., The pseudo-Newtonian force in time-varying spacetimes, Nuovo Cimento B 111 (1996), 79-84.

[43] Qadir A., Sharif M., General formula for the momentum imparted to test particles in arbitrary spacetimes, Phys. Lett. A 167 (1992), 331-334, gr-qc/0701098. 